\title{
RNA sequencing-based exploration of the effects of blue laser irradiation on mRNAs involved in functional metabolites of $D$. officinale
}

\author{
Hansheng $\mathrm{Li}^{1}$, Yuqiang Qiu ${ }^{2}$, Gang Sun ${ }^{\text {Corresp., }}{ }^{3}$, Wei Ye ${ }^{\text {Corresp. } 4}$ \\ ${ }^{1}$ College of Architectural Engineering, Sanming University, Sanming, Chian \\ 2 Xiamen Institute of Technology, Xiamen, China \\ 3 College of Resources and Chemical Engineering, Sanming University, Sanming, China \\ ${ }^{4}$ The Institute of Medicinal Plant, Sanming Academy of Agricultural Science, Shaxian, China \\ Corresponding Authors: Gang Sun, Wei Ye \\ Email address: sungang@nenu.edu.cn, yewei922@qq.com
}

Dendrobium officinale Kimura et Migo (D. officinale) has promising lung moisturizing, detoxifying, and immune boosting properties. Light is an important factor influencing functional metabolite synthesis in $D$. officinale. The mechanisms by which lasers affect plants are different from those of ordinary light sources; lasers can effectively address the shortcomings of ordinary light sources and have significant interactions with plants. Different light treatments (white, blue, blue laser) were applied, and the number of red leaves under blue laser was greater than that under blue and white light. RNA-seq technology was used to analyze differences in $D$. officinale under different light treatments. The results showed 465, 2107 and 1453 differentially expressed genes (DEGs) in LB-B, LB-W and W-B, respectively. GO, KEGG and other analyses of DEGs indicated that $D$. officinale has multiple blue laser response modes. Among them, the plasma membrane, cutin, suberine and wax biosynthesis, flavone and flavonol biosynthesis, heat shock proteins, etc. play central roles. Physiological and biochemical results verified that blue laser irradiation significantly increases POD, SOD, and PAL activities in $D$. officinale. The functional metabolite results showed that blue laser had the greatest promoting effect on total flavonoids, polysaccharides, and alkaloids. qPCR verification combined with other results suggested that $C R Y D A S H, S P A 1, H Y 5$, and PIF4 in the blue laser signal transduction pathway affect functional metabolite accumulation in $D$. officinale through positively regulated expression patterns, while $\mathrm{CO} 16$ and $M Y C 2$ exhibit negatively regulated expression patterns. These findings provide new ideas for the efficient production of metabolites in $D$. officinale. 
1 RNA sequencing-based exploration of the effects of blue laser irradiation on mRNAs involved in

2 functional metabolites of $D$. officinale

3 Hansheng $\mathrm{Li}^{1}$, Yuqiang Qiü ${ }^{2}$, Gang Sun ${ }^{3 *}$, Wei Ye ${ }^{*}$

41 College of Architectural Engineering, Sanming University, Sanming 365004, China;

52 Xiamen Institute of Technology, Xiamen 361021, China;

63 College of Resources and Chemical Engineering, Sanming University, Sanming 365004, China;

74 The Institute of Medicinal Plant, Sanming Academy of Agricultural Science, Shaxian 365509, China;

9 ABSTRACT

Dendrobium officinale Kimura et Migo (D. officinale) has promising lung moisturizing, detoxifying, and

immune boosting properties. Light is an important factor influencing functional metabolite synthesis in $D$.

officinale. The mechanisms by which lasers affect plants are different from those of ordinary light sources;

lasers can effectively address the shortcomings of ordinary light sources and have significant interactions with

plants. Different light treatments (white, blue, blue laser) were applied, and the number of red leaves under

blue laser was greater than that under blue and white light. RNA-seq technology was used to analyze

differences in D. officinale under different light treatments. The results showed 465, 2107 and 1453

differentially expressed genes (DEGs) in BL-B (blue laser versus blue light), BL-W (blue laser versus white

light) and W-B (white light versus blue light), respectively. GO, KEGG and other analyses of DEGs indicated

that $D$. officinale has multiple blue laser response modes. Among them, the plasma membrane, cutin, suberine and wax biosynthesis, flavone and flavonol biosynthesis, heat shock proteins, etc. play central roles. 
22

and PAL activities in D. officinale. The functional metabolite results showed that blue laser had the greatest promoting effect on total flavonoids, anthocyanin, polysaccharides, and alkaloids. qRT-PCR verification combined with other results suggested that CRY DASH, SPA1, HY5, and PIF4 in the blue laser signal transduction pathway affect functional metabolite accumulation in D. officinale through positively regulated expression patterns, while $C O 16$ and $M Y C 2$ exhibit negatively regulated expression patterns. These findings provide new ideas for the efficient production of metabolites in D. officinale.

Keywords Dendrobium officinale Kimura et Migo.; blue laser; functional metabolites; RNA-seq

\section{INTRODUCTION}

Dendrobium officinale Kimura et Migo is a medicinal plant of the Orchidaceae, Dendrobium genus. It can be called a "pulmonary scavenger" because it nourishes and regulates the lungs, produces fluids, moisturizes the lungs, detoxifies, and enhances immunity (Chen et al. 2018). In the fight against the novel coronavirus and the resulting pneumonia, D. officinale is highly favored by Chinese medicine experts. $D$. officinale is mainly distributed in western Fujian, eastern Zhejiang, southwest Anhui, Sichuan, northwest Guangxi and southeast Yunnan (Xu et al. 2015). D. officinale is susceptible to environmental influences such as light, temperature and humidity, and its natural survival rate is extremely low (Tang et al. 2019). After a long period of uncontrolled mining, wild resources of this species are becoming scarce (Tang et al. 2019). Modern chemical and pharmacological studies have shown that the main chemical components of $D$. officinale are polysaccharides, flavonoids, bibenzyls and alkaloids (Lin et al. 2019).

Researchers have used different methods to increase the content of functional metabolites in $D$. officinale, with light regulation being one of the most effective methods. Ordinary light sources refer to all light sources 
except lasers, including natural light and various artificial light sources (including LEDs). These all produce incoherent light. Additionally, there have been many reports on the study of plant functional metabolites. In a study examining the effect of different LED illumination modes on the accumulation of polysaccharides in $D$. officinale protocorms, it was found that mixed red and blue light had the best effects. Among the treatments, the yield of polysaccharides was the highest at the red light:blue light ratio of 1:3 (Lin et al. 2015). In research on $D$. officinale with different light qualities, it was found that monochromatic blue light within 15 days was beneficial for increasing the alkaloid content, and monochromatic yellow light within 30 days was beneficial for increasing the alkaloid content. After 30 days, treatment with a ratio of red to blue light of 2:3 was the most conducive to an increase in alkaloid contents (Liu et al. 2016).

To date, there have been few reports on the effects of laser light sources on plant functional metabolites. (1) The interaction between coherent light and plants and the difference between it and incoherent light is a research field with important theoretical value and high levels of innovation. The light emitted by ordinary light sources is composed of wave trains with a length of only a few microns (Tang et al. 2019; Luijtelaar et al. 2019). There is no correlation between the light wave trains, and the interaction between them and plant molecules is also irregular (Tang et al. 2019; Luijtelaar et al. 2019). Laser light sources are composed of several million to hundreds of millions of wave trains of light waves with a length of several meters to several kilometers (Tang et al. 2019; Luijtelaar et al. 2019). There is a strong correlation between the light wave trains. When they interact with plant molecules, they also show a certain regularity (Tang et al. 2019; Luijtelaar et al.

2019). (2) The mechanism of laser is completely different from ordinary light source, it not only can effectively solve the problems of ordinary light sources, but also has significant interaction with plants (Wan et al. 2020). (3) The laser light source plays an important role in the growth and development of plants. A large 
number of studies have found that pretreatment of seeds with laser light sources can significantly improve the growth, development and metabolism of a variety of plants ( $\mathrm{Li}$ et al. 2016). For example, when using a $4.0 \times 10^{-3} \mathrm{~J} / \mathrm{cm} 2 / \mathrm{s}$ laser to treat white lupin and broad bean, after $120 \mathrm{~h}$, it was found that the amylolytic enzyme activity in the seed cells reached the maximum value, the IAA content of the seeds increased, hypocotyl elongation accelerated, and the fresh weight and root length increased (Li et al. 2016). Research by Gao et al. (2016) found that a He-Ne laser can accelerate plant growth and development, possibly because it activates the synthesis and release of endogenous nitric oxide (NO) signal molecules and calcium signals in plant cells, which in turn mediates the occurrence of a series of intracellular signal cascade reaction pathways. In summary, the effect of laser light sources on the functional metabolites of $D$. officinale is worthy of in-depth study.

In the author's long-term research work on the interaction between ordinary light sources and plants (Li et al. 2019; Li et al. 2021), it was found that ordinary light sources may recognize and act on specific receptors on the cell wall (or cell membrane). The signal transduction pathway activates certain enzymes or substrate proteins, affects specific biochemical and metabolic processes in the cytoplasm, acts on nuclear genes, changes the expression patterns and transcriptional activities of related genes, translates to synthesize specific protein products, and regulates plant growth and development and secondary metabolism. However, the exact molecular mechanism by which lasers regulate plant functional metabolites is unclear. Such regulation may occur through a specific signal transduction pathway or may be caused by the electromagnetic effect of the laser or the energy conversion of laser irradiation or laser coherence (Li et al. 2016). These problems urgently need to be further explored and verified.

In this study, we used high-throughput sequencing technology to identify putative mRNAs and 
investigated their expression profiles in D. officinale under different light patterns. By comparing and analyzing the sequencing data of the treatment group and the control group, the difference between the functional metabolites of $D$. officinale under normal and laser light sources was found, the specific effects of the laser on mRNA and secondary metabolites of D. officinale were discovered, the secondary metabolites of D. officinale were mapped, and their signal transduction pathways were determined. These results will provide new ideas for the high-yield production of medicinal ingredients of D. officinale.

\section{MATERIALS AND METHODS}

\section{Plant materials and light treatments}

The $D$. officinale used in this study had 3 to 4 true leaves, a leaf width of approximately 2 to $3 \mathrm{~mm}$, and a seedling height of approximately $2 \mathrm{~cm}$. The following light and environmental parameters were used: blue laser $(450 \mathrm{~nm})$, blue light $(450 \mathrm{~nm})$, white light; total light intensity $\left(100 / \mathrm{umol}^{\circ} \mathrm{m}^{-2} \cdot \mathrm{S}^{-2}\right)$; light time $(12 \mathrm{~h} / \mathrm{d})$; humidity $55 \% \sim 60 \%$; and temperature $26-28{ }^{\circ} \mathrm{C}$. Tissue cultured seedlings of $D$. officinale were placed in a light incubator for 60 days. Tissue culture seedlings of $D$. officinale were maintained as previously described (Li et al. 2021). The control and light treatment samples were stored for subsequent nucleic acid extraction, high-throughput sequencing and functional metabolite content determination. White light was used as the control group, and the experimental group included blue light and blue laser treatments. Samples treated with blue laser versus blue light, blue laser versus white light and blue light versus white light were named BL-B, $\mathrm{BL}-\mathrm{W}$, and B-W, respectively.

\section{mRNA library construction and Illumina HiSeq sequencing}

In this study, high-throughput sequencing was performed on samples subjected to three treatments - white light, 
106

107

108

109

110

111

112

113

114

115

116

117

118

119

120

121

122

123

124

125

126

blue light, and blue laser irradiation - with 3 biological replicates for each group. RNA quantification and qualification were performed according to the method of Li et al (2019 and 2021). Sequencing library preparation and high-throughput sequencing were subsequently performed using the Illumina HiSeq platform (Beijing, China). All sequencing data of $D$. officinale under the different light treatments were deposited in the National Genomics Data Center (NGDC) Sequence Read Archive (accession number PRJCA006154).

The adaptor sequences and low-quality sequence reads were removed from the data sets. Raw sequences were transformed into clean reads after data processing. These clean reads were then mapped to the reference genome sequence (Zhang et al. 2016). The reference genome version of D. officinale in this manuscript was updated on April 11, 2019 (Li et al. 2021). Only reads with a perfect match were further analyzed and annotated based on the reference genome.

\section{Gene functional annotation and differential expression analysis}

Gene function was annotated as previously described (Li et al. 2021). Differential expression analysis of two conditions/groups was performed using the DESeq R package (1.10.1). DESeq provides statistical tools for determining differential gene expression using a model based on the negative binomial distribution. The resulting P-values were adjusted using Benjamini and Hochberg's approach for controlling the false discovery rate (Benjamini et al. 2005). Genes with an adjusted $\mathrm{P}$-value $<0.01$ and absolute value of $\log _{2}$ (Fold change) $>1$ found by DESeq were assigned as differentially expressed.

\section{GO and KEGG enrichment analyses of differentially expressed genes}

Gene Ontology (GO) enrichment analysis of the differentially expressed genes (DEGs) was implemented by the GOseq R package-based Wallenius noncentral hypergeometric distribution (Young et al. 2010), which can adjust for gene length bias in DEGs. KEGG (Kyoto Encyclopedia of Genes and Genomes) enrichment analysis 
127

128

129

130

131

132

133

134

135

136

137

138

139

140

141

142

143

144

145

146

147

of the DEGs was performed using KOBAS (Chen et al. 2011) software.

\section{Determination of functional metabolites}

The polysaccharide, flavonoid and alkaloid contents in the D. officinale stems and leaves were determined as previously described (Li et al. 2021).

The anthocyanin of $D$. officinale stems and leaves were determined by the method described by Chen et al. (2017). Two grams of Freeze-dried grains was weighed and added to $20 \mathrm{~mL}$ of extraction solution (1\% vanillin methanol solution: $15 \%$ methyl hydrochloride alcohol solution $=1: 1)$. Then dilute the volume to $50 \mathrm{~mL}$ with the extraction solution and let it stand for $2 \mathrm{~h}$. Take $1 \mathrm{~mL}$ of supernatant in a $250 \mathrm{~mL}$ erlenmeyer flask, add 100 $\mathrm{mL}$ of $1 \%$ hydrochloric acid solution, and water bath for $10 \operatorname{mins}\left(60^{\circ} \mathrm{C}\right)$. The absorbance was measured with a UV-visible spectrophotometer, and the wavelengths of anthocyanins were measured at $520 \mathrm{~nm}$. The anthocyanin contents in the $D$. officinale stems and leaves were calculated according to established standard curves.

\section{Determination of enzyme activity}

The leaves of $D$. officinale were ground with liquid nitrogen, $0.2 \mathrm{~g}$ grains were weighed, and $1 \mathrm{ml}$ extract was added. Then, the extract was centrifuged $\left(4^{\circ} \mathrm{C}, 8000 \mathrm{~g}, 10 \mathrm{~min}\right)$, and the supernatant was collected and placed on ice for testing. The supernatant was collected for enzyme activity assays. Superoxide dismutase (SOD), peroxidase (POD) and Phenylalaninammonialyase (PAL) were assayed using commercial kits (Suzhou Keming Biotechnology Limited Company, Suzhou, China) and a DU640 spectrophotometer (Beckman, Brea, CA, USA) according to the manufacturers' instructions and a previous report.

\section{Quantitative real-time PCR (qRT-PCR) analysis}

Total RNA from $D$. officinale leaves was used for qRT-PCR validation of mRNAs. Twelve DEGs were 
148

subjected to qRT-PCR analysis on a LightCycler 480 Real-Time PCR System (Roche, Switzerland). cDNA synthesis, the reaction system and the procedures, etc. referred a previous method ( $\mathrm{Li}$ et al. 2021). The relative mRNA expression levels were calculated using the comparative $2^{-\Delta \Delta \mathrm{Ct}}$ method. The $A C T I N$ gene was used as the reference gene (Shen et al. 2017). The primer sequences are listed in Supplementary Table S1.

\section{Data analysis}

The quantitative results of gene expression, antioxidant enzyme activity and functional metabolites of $D$. officinale were determined with at least 3 biological replicates. Using SPSS V 19.0 and one-way analysis of variance (ANOVA) with Duncan's tests, the impact of different illumination modes on the indicators of $D$. officinale was analyzed. GraphPad Prism 6.0 software and OmicShare online software were used for drawing.

\section{RESULTS}

\section{Growth state of $D$. officinale under different light treatments}

Lasers can affect the growth state of $D$. officinale. The results of this study found that there were no significant differences in plant height, stalk thickness, and leaf area between white light, blue light and blue laser, but the number of red leaves under the blue laser treatment was greater than that under the blue and white light treatments (Figures 1A-F; Table S2).

\section{Sequencing and assembly of transcriptomic data}

To study the effects of lasers on functional metabolite-related genes of $D$. officinale, 9 mRNA libraries were constructed and sequenced in this study (Table 1). After removing the linker sequences, the RNA-seq data of $D$. officinale under different light treatments produced $40,640,606$ to $54,223,452$ reads. A total of $87.96 \%-88.97 \%$ of clean reads could be completely matched to the reference genome of D. officinale, while $85.70 \%-86.70 \%$ of 
169

170

171

172

173

174

175

176

177

178

179

180

181

182

183

184

185

186

187

188

189

clean reads could be matched to a single site in the reference genome of $D$. officinale, indicating that sequencing reads matched the reference genome of $D$. officinale to a high degree. The Q30 values of $D$. officinale samples were all higher than $92.47 \%$, indicating the high reliability of the $D$. officinale transcriptome sequencing data.

\section{Analysis of differentially expressed genes under different light treatments}

This study analyzed the results of transcriptome sequencing and discovered 3735 new genes, 2888 that were functionally annotated, and 2500 DEGs (Table S3). To study the gene expression of D. officinale, this study divided all genes into three categories, namely, high expression (FRKM $>50)$, medium expression ( $5 \leq$ FRKM $\leq 50)$ and low expression (FRKM $<5)$. The results showed that most genes were in the low and medium expression groups, while a few genes were highly expressed (Figure 2A).

To study the DEGs of D. officinale, we drew Venn diagrams of DEGs under different combinations. The results showed that 465 DEGs were identified in the BL-B combination, 2107 were identified in the BL-W combination, and 1453 were identified in the B-W combination. Eighty genes were jointly regulated by the three combinations of BL-B, BL-W and B-W; 141 genes were jointly regulated by the two combinations of BL-B and BL-W; 325 genes were jointly regulated by the two combinations of BL-B and B-W; and 1139 genes were jointly regulated by the two combinations of BL-W and B-W. Seventy-nine genes were specifically regulated by the BL-B combination, 253 genes were specifically regulated by the BL-W combination, and 723 genes were specifically regulated by the B-W combination (Figure 2B). Cluster analysis showed that 2500 DEGs could be divided into six expression patterns under different light treatments (Figure 2C).

\section{GO analysis of DEGs in D. officinale}

To further understand the influence of laser irradiation on D. officinale, GO enrichment analysis was 
190

191

192

193

194

195

196

197

198

199

200

201

202

203

204

205

206

207

208

209

210

performed on the DEGs in the three comparisons of BL-B, BL-W, and B-W (Table 2).

For the biological process category in the GO analysis, the BL-B-specific terms included SRP-dependent

cotranslational protein targeting to membrane, regulation of translational initiation, regulation of protein

complex assembly, response to endoplasmic reticulum stress, etc. The BL-W-specific biological processes

included jasmonic acid metabolic process and oxylipin biosynthetic process.

For the cell component category in the GO analysis, the BL-B-specific terms included plasma membrane part, vacuole, and replisome, and only cell wall was specific to BL-W.

For the molecular function category in the GO analysis, the BL-B-specific terms included unfolded protein binding and RNA-directed DNA polymerase activity.

In summary, the effect of laser irradiation on the functional metabolites of $D$. officinale might be closely related to SRP-dependent cotranslational protein targeting to the membrane, jasmonic acid metabolic process, oxylipin biosynthetic process, plasma membrane, cell wall, etc.

\section{KEGG enrichment analysis of DEGs in D. officinale}

This study included KEGG enrichment analysis of the identified DEGs (Figure 3). The top 5 enriched pathways were cutin, suberine and wax biosynthesis, vitamin B6 metabolism, photosynthesis-antenna proteins, flavone and flavonol biosynthesis, and diterpenoid biosynthesis for the BL-B combination (Figure 3A). The top 5 enriched pathways were plant hormone signal transduction, flavonol and flavonol biosynthesis, vitamin B6 metabolism, cutin, suberine and wax biosynthesis, and circuitadian rhythm-plant for the BL-W combination (Figure 3B). The top 5 enriched pathways were flavone and flavonol biosynthesis, flavonoid biosynthesis, stilbenoid, diarylheptanoid and gingerol biosynthesis, monobactam biosynthesis, and alphalinolenic acid metabolism for the B-W combination (Figure 3C). 

signal transduction, and alpha-linolenic acid metabolism were among the top 20 most enriched pathways in the

plays an important role in these pathways. white light.

The top 10 up- and downregulated DEGs under different light treatments 
232 ABCG11) and polysaccharide metabolism and synthesis genes (INV*DC4, CSLA9) in the LB-B combination

233 (Table 3). The top 10 downregulated genes mainly included chlorophyll synthesis-related genes (CAB3C),

234

235

236

237

238

239

240

241

242

243

244

245

246

247

248

249

250

251

252

isoflavone synthesis-related genes (CYP81E1, CYP81E1), and serine-related proteases (HT1, SAT2) in the

LB-B combination (Table 3). The top 10 upregulated genes mainly included early light-inducible protein (ELIP1), transcription factor (MYB114), polysaccharide metabolism synthesis gene (MAN6), and heat shock protein (HSP83A, HSP18.6) in the LB-W combination (Table 4). The top 10 downregulated genes mainly included cytochrome-related genes (CYP94A1), ethylene-responsive transcription factors (ERF112, ERF114), and isoflavone synthesis-related genes (CYP81E1) in the LB-W combination (Table 4).

\section{Levels of physiological and biochemical indicators in D. officinale under different light treatments}

In this study, the physiological and biochemical indexes of $D$. officinale leaves were measured under different light treatments, and the results are shown in Figure 4. The POD enzyme activity value under the blue laser was $1866.67 \mathrm{U} \mathrm{g}^{-1}$, blue light was $1713.33 \mathrm{U} \mathrm{g}^{-1}$, and white light was $760.00 \mathrm{U} \mathrm{g}^{-1}$ (Figure 4A; Table S5). The SOD enzyme activity value under blue laser treatment was $50.15 \mathrm{U} \mathrm{g}^{-1}$, blue light was $18.90 \mathrm{U} \mathrm{g}^{-1}$, and white light was $8.49 \mathrm{U} \mathrm{g}^{-1}$ (Figure 4B; Table S6). The PAL enzyme activity under the blue laser treatment was 182.89 $\mathrm{U} \mathrm{g}^{-1}$, blue light was $173.03 \mathrm{U} \mathrm{g}^{-1}$, and white light was $50.29 \mathrm{U} \mathrm{g}^{-1}$ (Figure 4C; Table S7). The activities of POD, SOD and PAL in D. officinale were the highest under the blue laser treatment, followed by blue light, and the lowest under white light. Therefore, blue laser is most beneficial for promoting the activities of POD, SOD and PAL in D. officinale.

\section{Secondary metabolite contents in D. officinale under different light treatments}

In this study, the functional metabolites of $D$. officinale leaves and stems were measured under different light treatments, and the results are shown in Figure 5. Among the differentially expressed flavonoid metabolic 
253

254

255

256

257

258

259

260

261

262

263

264

265

266

267

268

269

270

271

272

273

pathway synthesis genes, the expression levels of most genes under blue laser treatment were higher than those under the other treatments (Figure 5A). The flavonoid content of D. officinale leaves under blue laser irradiation was the highest at $73.11 \mathrm{mg} / \mathrm{g}$, followed by that under blue light at $52.55 \mathrm{mg} / \mathrm{g}$ and white light at $29.90 \mathrm{mg} / \mathrm{g}$ (Figure 5B; Table S8). The flavonoid content of D. officinale stems under blue laser irradiation was the highest at $41.38 \mathrm{mg} / \mathrm{g}$, followed by blue light at $30.83 \mathrm{mg} / \mathrm{g}$ and white light at $22.60 \mathrm{mg} / \mathrm{g}$ (Figure $5 \mathrm{C}$;

Table S9). Among the differentially expressed anthocyanin metabolic pathway synthesis genes, the expression levels of most genes under blue laser treatment were higher than those under the other treatments (Figure $5 \mathrm{~A}$ ).

The anthocyanin content of $D$. officinale leaves under blue laser irradiation was the highest at $20.66 \mathrm{mg} / \mathrm{g}$, followed by that under blue light at $16.82 \mathrm{mg} / \mathrm{g}$ and white light at $15.07 \mathrm{mg} / \mathrm{g}$ (Figure 5D; Table S10). The flavonoid content of $D$. officinale stems under blue laser irradiation was the highest at $9.23 \mathrm{mg} / \mathrm{g}$, followed by blue light at $9.17 \mathrm{mg} / \mathrm{g}$ and white light at $8.43 \mathrm{mg} / \mathrm{g}$ (Figure 5E; Table S11). Among the differentially expressed synthetic genes in the polysaccharide metabolic pathway, the expression levels of all genes under the blue laser treatment were higher than those under the other treatments (Figure 5F). The leaves and stems had the highest polysaccharide contents under blue laser treatment, followed by blue light, and white light resulted in the lowest polysaccharide content (Figure 5G-H; Table S12-S13). Among the differentially expressed synthetic genes in the alkaloid metabolic pathway, the expression of $P P O$ and $T T A$ genes under blue laser treatment was higher than that under other treatments (Figure 5I). The leaf alkaloid content was the highest under blue laser treatment at $73.04 \mathrm{mg} / \mathrm{g}$, that under blue light treatment was $72.55 \mathrm{mg} / \mathrm{g}$, and that under white light treatment was the lowest (Figure 5J; Table S14). The highest alkaloid content of stems under blue laser treatment was $34.29 \mathrm{mg} / \mathrm{g}$, under blue light treatment the content was $34.62 \mathrm{mg} / \mathrm{g}$, and under white light treatment the contents were the lowest (Figure 5K; Table S15). Therefore, compared with ordinary light 
274

275

276

277

278

279

280

281

282

283

284

285

286

287

288

289

290

291

292

293

294

sources, blue lasers can significantly increase the contents of total flavonoids, polysaccharides, and alkaloids in D. officinale.

\section{Identification of DEGs in D. officinale under different light treatments by qRT-PCR}

In this study, 9 groups of mRNAs were verified by qRT-PCR, and the results are shown in Figure 6 . Some heat shock proteins, cutin, suberine and wax biosynthesis, and hormone signal transduction pathway-related genes play an important role in blue laser-mediated regulation of functional metabolites of $D$. officinale. For example, the expression level of HSP70 was highest under blue laser treatment, followed by white light, and was the lowest under blue light (Figure 6A; Table S14). The expression levels of CYP86A4S and ERF13 were the highest under blue laser irradiation, followed by blue light and white light (Figure 6B-C; Table S14). Blue laser signal transduction pathway genes are closely related to the synthesis of functional metabolites of $D$. officinale. For example, the expression levels of $C R Y D A S H, S P A 1$, and HY5 were the highest under blue laser irradiation, followed by blue light, and white light (Figure 6D-F; Table S14). The expression levels of CO16 and MYC2 were highest under white light, followed by blue light, and blue laser light had the lowest expression (Figure 6G-H; Table S14). The expression level of PIF4 was highest under blue laser treatment, followed by white light, and was the lowest under blue light (Figure 6I; Table S14). Therefore, $C R Y D A S H$, SPA1, HY5, and PIF4 in the blue laser signal transduction pathway might affect the accumulation of functional metabolites of $D$. officinale through positively regulated expression patterns, while CO16 and MYC2 exhibit negatively regulated expression patterns.

\section{DISCUSSION}

Cell Signaling Perception and Conduction in D. officinale under Blue Laser 
295 GO analysis identified SRP-dependent cotranslational protein targeting the membrane, regulation of

296

297

298

299

300

301

302

303

304

305

306

307

308

309

310

311

312

313

314

translational initiation, regulation of protein complex assembly, response to endoplasmic reticulum stress, plasma membrane part, and vacuole as significantly enriched in only the LB-B combination (Table 2). Oxylipin biosynthetic process and the cell wall were significantly enriched in only the LB-W combination (Table 2). All of the above pathways involve cell signaling perception and conduction in D. officinale under blue laser treatment.

When $D$. officinale responds to a blue laser, the cell wall composition changes accordingly. The sensor elements of external factors are mainly distributed between the cell wall and the cell membrane, leading to an increase in the concentration of $\mathrm{Ca}^{2+}$ in the cytosol (Hamann et al. 2015). The cell membrane is a barrier that prevents external substances from freely entering the cell and is the site of information, energy, and material exchange between external factors and the cell (Morales-Cedillo et al. 2015). The cell membrane maintains the stability of the intracellular environment so that the physiological metabolic pathways in the plant proceed in an orderly manner. The endoplasmic reticulum is the cytoplasmic membrane system and is connected to the cell membrane on the outside and communicates with the outer membrane of the nuclear membrane on the inside. The endoplasmic reticulum organically connects the various structures in the cell into a whole, effectively increases the membrane area in the cell, and plays the role in external signal transmission and intracellular material transport (Wang et al. 2017). Ribosomes are attached to the rough endoplasmic reticulum, and their arrangement is relatively smooth. The endoplasmic reticulum is smooth, and its function is to synthesize protein macromolecules and transport them out of the cell or to other parts in the cell. Oxylipin is a metabolite of oxidized fatty acids and their derivatives (Kriechbaumer et al. 2020). Such substances are found in bacteria, fungi, algae, and flowering plants. Oxylipin is a signaling molecule that regulates plant growth and 
development and plays an important role in responding to external factors (Satoh et al. 2014; Savchenko et al.

$3172014)$

Therefore, when the cell wall of D. officinale is affected by blue laser, the composition and structure of

the cell wall dynamically changes to maintain the integrity of the cell wall and adapt to cell growth. The

protein on the cell wall and the outer side of the membrane is the first sensor element to sense the blue laser.

Together with the receptor protein, these sensing elements initiate a blue laser response, transmit information

to the endoplasmic reticulum of $D$. officinale through a signal cascade, and then regulate the expression of cell

wall components and extracellular proteins through a feedback mechanism.

\section{D. officinale responds to blue lasers through cutin, suberine and wax biosynthesis}

This study found that cutin, suberine and wax biosynthesis was among the top 20 most enriched pathways for the three combinations (LB-B, LB-W, B-W) by KEGG analysis (Figure 3). The stratum corneum is a lipid water-retaining layer formed on the outer surface of the epidermal cell wall of terrestrial plants. The basic function of the stratum corneum is to retain water, and it also plays a role in external factor response, selfcleaning, and organ development (Ingram et al. 2017). The stratum corneum is usually composed of cutin and wax. Cutin is the main structural component of the stratum corneum, and its main component is polyester. The waxy components are mainly very long-chain saturated fatty acids and their derivatives, as well as flavonoids and triterpenoids (Duan et al. 2017). These components are synthesized on the endoplasmic reticulum and transported to the cell surface to further form a complete stratum corneum structure. Relevant studies have found that plant epidermal wax reflects ultraviolet radiation and visible light, and wax most strongly reflects ultraviolet radiation than visible light (Bruhn et al. 2014). Some studies also found that compared with normal plants, corn lacking wax in the epidermis suffered more damage from ultraviolet rays, and leaf shape and plant 
337

338

339

340

341

342

343

344

345

346

347

348

349

350

351

352

353

354

355

356

357

genetics were significantly affected (Duan et al. 2017). Compared with leaves with relatively less epidermal

wax, leaves with more epidermal wax absorbed more ultraviolet rays, with reduced damage to the plant

(Vishwanath et al. 2015). In addition, suberin is a glycerol-phenol-lipid polymer whose composition is similar

to that of cell wall wax. Suberin can control the outflow of water and solutes, and it can also play an important

role in the resistance of plants to external factors such as drought, salt and strong light (Vishwanath et al. 2015;

Martins et al. 2014). Therefore, long-term cultivation of $D$. officinale under an appropriate amount of blue

laser (strong radiation energy) promotes the formation of plant wax, cutin, and cork, ensures the normal growth

and development of plants, and increases the accumulation of functional metabolites.

\section{Heat shock proteins play an important role in the response of $D$. officinale to blue lasers}

This study found that the top 10 differentially expressed genes in the LB-B and LB-W combinations involved heat shock protein-related genes, including HSP70, HSP23, HSP18.6, HSP83A, CCOMT, and ABCG11

(Table 3-4). This study also found that the activities of POD and SOD were higher under blue laser than under blue and white light (Figure 4A-4B). GO analysis identified the oxidoreductase activity, acting on single donors with incorporation of molecular oxygen, incorporation of two atoms of oxygen and oxidoreductase activity, acting on paired donors, with incorporation or reduction of molecular oxygen as significantly enriched in three combination (Table 2). Heat shock proteins play an important role in the growth of plants, as both essential and defensive proteins, helping to rebuild the normal structure and function of cells (Jacob et al. 2017). When plants are under adverse stress, heat shock proteins mainly participate in the defencse response in the following ways. (1) Heat shock proteins can protect protein functional structure, prevent abnormal protein aggregation, and remove potentially harmful denatured proteins (Jacob et al. 2017; Fu et al. 2015). (2) Heat shock proteins maintain membrane integrity by increasing 
358

359

360

361

362

363

364

365

366

367

368

369

370

371

372

373

374

375

376

377

378

membrane lipid order and reducing membrane lipid fluidity (Jacob et al. 2017; Fu et al. 2015). (3) Heat shock

proteins act as antioxidants to remove excess reactive oxygen species (ROS). Plants have a complete internal

protective enzyme system to remove the damage caused by ROS, thereby maintaining the normal functioning

of plant cells. Plant cells mainly use enzymatic and nonenzymatic antioxidant systems to reduce oxidative

damage. The compounds in the enzymatic system include superoxide dismutase (SOD), peroxidase (POD),

and catalase (CAT), etc (Luo et al. 2020). Compounds in the nonenzymatic system include flavonoids,

ascorbate, glutathione, etc (Luo et al. 2020). The increase in plant ROS levels can cause oxidative stress, which

in turn causes oxidative damage to nucleic acids, proteins, carbohydrates and lipids. Heat shock proteins may

increase glutathione by enhancing the activity of glucose-6-phosphate dehydrogenase (G6PD). The reduction

of peptides (glutathione, GSH) eliminates excess ROS (Liu et al. 2016). Therefore, heat shock proteins might

play an important role in the response of D. officinale to blue lasers.

\section{Some functional metabolic pathways are involved in the effect of blue laser irradiation on $D$. officinale}

This study found that the PAL enzyme activity under blue laser treatment was significantly higher than that

under blue and white light treatments (Figure 4C). PAL is the key and rate-limiting enzyme in the phenylpropane metabolic pathway. Phenylalanine is converted into cinnamic acid under the catalysis of the

PAL enzyme. An increase in its activity can produce lignin, cork, flavonoids and anthocyanins, thereby improving the ability to deal with external factors. In the D. officinale response to blue laser irradiation, this study also found that phenylalanine metabolism, flavone and flavonol biosynthesis, starch and sucrose metabolism, fructose and mannose metabolism, tropane, piperidine and pyridine alkaloid biosynthesis and other pathways were among the top 20 most enriched pathways (Figure 3), and these pathways are closely related to the biosynthesis of flavonoids, polysaccharides, and alkaloids. The above results indicate that the 
379

381

blue laser promotes the accumulation of total flavonoids, polysaccharides and alkaloids in D. officinale.

Light is an indispensable factor for plant growth and development. Plants form their own unique metabolic basis by sensing different light signals (Nhut et al. 2015). This study found that the PAL enzyme activity under blue laser treatment was significantly higher than that under blue and white light treatments. Flavonoids in plants have the protective function of scavenging ROS, and light will cause different degrees of photooxidative stress, thereby inducing the ROS scavenging mechanism and affecting the accumulation of flavonoids (Lan et al. 2017; Martin et al. 2015). This effect may have been due to the high-energy blue laser used, which caused greater photooxidative damage to D. officinale, and flavonoids played a role in protecting plants by removing ROS. The synthesis of plant alkaloids is mainly affected by genetic factors and the external environment (Wang et al. 2010). The external factors that affect the synthesis of plant alkaloids mainly include strong and weak light stress, temperature and drought (Wang et al. 2010). Light can promote an increase in secondary messengers (calmodulin, G protein and cAMP contents), which in turn activates the phytochrome and cryptochrome system and enables the expression of downstream genes related to alkaloid metabolism in plants (Wang et al. 2010). In this study, the blue laser may promote an increase in the secondary messenger content, thereby increasing their contents in $D$. officinale. Plants can survive under adverse conditions such as ultraviolet light, strong light, low temperature, and high salt because they secrete long-chain polysaccharides, that is, extracellular polysaccharides (EPSs), outside the cell during growth and metabolism. On the one hand, EPSs play a role as osmotic regulators; on the other hand, they can form a protective film on the cell surface to protect proteins from inactivation (Chang et al. 2020). In summary, the metabolism of flavonoids, alkaloids, and polysaccharides plays an important role in the response of $D$. officinale to blue lasers.

The blue laser signal transduction pathway affects the accumulation of functional metabolites in $D$. 
400

401

402

403

404

405

406

407

408

409

410

411

412

413

414

415

416

417

418

419

420

\section{officinale}

This study showed that blue laser irradiation was the most beneficial among the treatments to promote the accumulation of total flavonoids, polysaccharides, and alkaloids in D. officinale. Based on the study of the blue light signal transduction pathways in model plants such as Arabidopsis (Meng et al. 2013; Liu et al. 2013;

Wang et al. 2015; Yang et al. 2017), transcription was explored using D. officinale data, the blue laser signal network-related genes were screened, and the blue laser signal network affecting the functional metabolites of D. officinale was initially constructed (Figure 7A).

Plants can accurately perceive light conditions from UV-B to far-red light through a variety of photoreceptors ( $\mathrm{Su}$ et al. 2017). The response of plants to blue light is mainly mediated by CRY photoreceptors, including $C R Y 1, C R Y 2$ and $C R Y-D A S H$ (Yang et al. 2017). In this study, only CRYDASH was found among the DEGs, and the blue laser treatment of Dendrobium_catenatum_newGene_8958 and geneMA16_Dca024302 (CRY DASH) had the highest expression level, followed by blue light treatment and white light (Figure 7B). The CRY DASH protein has the biochemical activity of repairing single-stranded DNA and may be involved in the protection of organelle genes. It can directly bind DNA or RNA and can regulate the developmental process by regulating gene transcription, but its signal transduction pathway has not been reported thus far (Castrillo et al. 2015). Therefore, it is speculated that the CRY-DASH receptor plays a leading role in the response of $D$. officinale to blue laser irradiation.

In the plant COP1/SPA pathway, CRYs can interact with the SPA1/COP1 complex at the posttranscriptional level to indirectly regulate gene expression (Wang et al. 2015). CRY1 inhibits the degradation of HY5 (long hypocotyl 5) and HFR1 (long hypocotyl in Far-Red1) proteins by COP1 under blue light (Yang et al. 2017). CRY2 can also inhibit the degradation of the transcription factor CO protein by COP1 
421 under blue light. These transcription factors regulate the photomorphogenesis of plants (Yang et al. 2017). In

422 this study, only three of these genes, SPA1, HY5, and CO, were found among the DEGs. Blue laser processing

gene-MA16_Dca007860 (SPA1), Dendrobium_catenatum_newGene_1002 and gene-MA16_Dca018475 (HY5)

had the highest expression, followed by blue laser processing, followed by white light, and gene-

MA16_Dca001117 (CO5), gene-MA16_Dca019470 (CO14), gene-MA16_Dca019470 (CO14), and gene-

MA16_Dca024960 (CO16) were the opposite (Figure 7B). Under light conditions, COP1 and SPA can form

a complex in the nucleus and regulate the synthesis of plant functional metabolites (Huang et al. 2014). HY5

(LONG HYPOCOTYL 5), as a key factor of light signal transduction, also plays an important role in the

regulation of plant functional metabolites (Shi et al. 2016). Studies in Arabidopsis have confirmed that blue

light can regulate the expression of PAP1 (an R2R3-MYB transcription factor) through the light signal

transcription factor HY5 and thereby regulate the metabolic synthesis of flavonoids (Shi et al. 2016). Therefore,

it is speculated that SPA1 and HY5 play a positive role in the blue laser-mediated regulation of functional

metabolites in D. officinale, while CO plays a negative regulatory role.

In the plant PIF pathway, PIFs (phytochrome-interacting factors) play a key regulatory role in the light signaling pathway (Liu et al. 2019). PIFs can transduce light signals downstream of phytochrome and 
442

443

444 transgenic plants. This showed that AaPIF3 plays a significant role in regulating the biosynthesis of artemisinin (Liu et al. 2015). Arabidopsis PIF3 can positively regulate anthocyanin biosynthesis, while PIF4 and PIF5 play a negative regulatory role in red light-induced anthocyanin accumulation. Relevant studies have found that red light effectively increased anthocyanin accumulation in wild-type plants, while the effect in pif4 and pif5 mutant plants was significantly enhanced, and the effect in PIF4OX and PIF5OX overexpression plants was significantly weakened. The transcription levels of the anthocyanin synthesis-related genes $C H S$, $F 3^{\prime} H, D F R, L D O X, P A P 1$ and TT8 in pif4- and pif5-mutant plants were significantly increased, while those in PIF4OX- and PIF5OX-overexpressing plants were the opposite (Zhang et al. 2019). The blue laser treatment increased expression of Dendrobium_catenatum_newGene_10821 (PIF3) and gene-MA16_Dca021603 (PIF4), while it was lowest under the white light treatment (Figure 7B). Therefore, it is speculated that PIF3 and PIF4 play an important role in the regulation of functional metabolites of $D$. officinale by blue laser irradiation.

In the plant MYC2 pathway, MYC2 (a basic helix-loop-helix (bHLH) transcription factor) is a node in the blue light signaling pathway regulating plant metabolism and metabolite synthesis (Sethi et al. 2014). In research on Catharanthus roseus, it was found that $M Y C 2$ can promote the accumulation of alkaloids (Gangappa et al. 2013). Arabidopsis MYC2 positively regulates the biosynthesis of flavonoids by positively regulating other transcription factors. In contrast, $M Y C 2$ negatively regulates the biosynthesis of the JAresponsive tryptophan derivative indole glucosinolates (Zhang et al. 2013). In this study, only the $M Y C 2$ gene was found among the DEGs, and the MA16_Dca025360 and MA16_Dca006997 (MYC2) genes had the highest expression levels under white light, followed by blue light and blue laser light (Figure 7B). Therefore, it is speculated that $M Y C 2$ is a negative regulator of the blue laser irradiation effects on the functional metabolites of $D$. officinale.

PeerJ reviewing PDF | (2021:07:63846:1:1:NEW 20 Nov 2021) 
In summary, this study suggests that blue laser can affect the synthesis of functional metabolites of $D$.

464

officinale through three ways. First, under the action of a blue laser, the interaction between CRYs and the SPA protein inhibits the activation of COP1 by SPA, thereby inhibiting the degradation of HY5 and CO transcription factors by COP1 and upregulating the expression of light-regulated genes, thereby promoting the synthesis of functional metabolites in D. officinale. Second, the blue laser negatively regulates the synthesis of functional metabolites through the transcription factor MYC2. Third, CRYs bind to PIF3 and PIF4, thereby regulating the synthesis of functional metabolites.

\section{CONCLUSION}

This study provides the first demonstration of blue laser irradiation on mRNAs involved in functional metabolites of $D$. officinale through an RNA-seq analysis. We found that the number of red leaves of $D$. officinale under blue laser was greater than that under blue and white light. Blue laser had the greatest promoting effect on total flavonoids, anthocyanin, polysaccharides, and alkaloids. Based on the transcriptomic, physiological and biochemical analyses, we revealed that $D$. officinale responds to blue lasers through cutin, suberine and wax biosynthesis. Heat shock proteins play an important role in the response of $D$. officinale to blue lasers. The blue laser signal transduction pathway affects the accumulation of functional metabolites in $D$. officinale. These findings will be helpful for generating new insights for the high-yield production of functional metabolites of D. officinale.

\section{Data Availability}

The following information was supplied regarding data availability: Raw data are available as a Supplementary 
484 File.

485

486

487

490

491

492

493

494

495

496

500

501

502

503

504

Supplementary Materials Table S1: Primers information used for real-time PCR analysis of $D$. officinale genes; Table S2: Phenotype of $D$. officinale after different light treatments; Table S3: The related genes of $D$. officinale under different light treatments; Table S4: The FPKM value of differentially expressed genes of $D$. officinale under different light treatments; Table S5: POD activity of leaves in D. officinale under different light treatments; Table S6: SOD activity of leaves in D. officinale under different light treatments; Table S7:

PAL activity of leaves in D. officinale under different light treatments; Table S8. Flavonoid contents of leaves in D. officinale under different light treatments; Table S9. Flavonoid contents of stems in D. officinale under different light treatments; Table S10 Anthocyanin contents of leaves in D. officinale under different light treatments; Table S11 Anthocyanin contents of stems in D. officinale under different light treatments; Table S12: polysaccharide contents of leaves in D. officinale under different light treatments; Table S13: polysaccharide contents of stems in D. officinale under different light treatments; Table S14: Alkaloid contents of leaves in D. officinale under different light treatments; Table S15: Alkaloid contents of stems in $D$. officinale under different light treatments; Table S16: The Ct mean of DEGs in D. officinale under different light treatments; Table S17: The relative expression of DEGS in D. officinale under different light treatments.

Author contribution statement Hansheng Li performed the experiments, analyzed the data, prepared figures and/or tables, authored or reviewed drafts of the paper, and approved the final draft. Peiyu Wang performed the experiments, analyzed the data, authored or reviewed drafts of the paper, and approved the final draft. Yaqian Wang analyzed the data, authored or reviewed drafts of the paper, and approved the final draft. Yan 
505 Fang analyzed the data, authored or reviewed drafts of the paper, and approved the final draft. Wei Ye and

506 Gang Sun conceived and designed the experiments, analyzed the data, prepared figures and/or tables, authored

507 or reviewed drafts of the paper, and approved the final draft.

508

509

Acknowledgments This work was funded by the National Natural Science Foundation of China (31501802),

the Natural Science Foundation of Fujian Province (2020J01377), the Education research project for young

and middle-aged teachers in Fujian (JAT190696), the Sanming University Scientific Research Foundation for

High-level Talent (18YG01, 18YG02, 19YG06), and the 2019 and 2020 Special Commissioner of Science and

Technology of Fujian Province. We thank American Journal Experts for editing the English text of a draft of this manuscript.

515

Conflicts of Interest The authors have no conflict of interest to declare.

\section{REFERENCES}

1. Benjamini Y, Yekutieli D. 2005. Quantitative trait loci analysis using the false discovery rate. Genetics 171(2):783-790.

2. Bruhn D, Mikkelsen T N, Rolsted M, Egsgaard H, Ambus P, Papen H. 2014. Leaf surface wax is a source of plant methane formation under UV radiation and in the presence of oxygen. Plant Biology 16(2):512-516.

3. Bruhn D, Mikkelsen T N, Rolsted M, Egsgaard H, Ambus P. 2014. Leaf surface wax is a source of plant methane formation under UV radiation and in the presence of oxygen. Plant Biology, 16(2):512-516. 
4. Castrillo M, Bernhardt A, Valos J, Batschauer A, Pokorny R. 2015. Biochemical characterization of the DASH-type cryptochrome CryD from Fusarium fujikuroi. Photochemistry \& Photobiology 91(6): $1356-1367$.

5. Chang XG, Chen XF,Gong P. 2020. Antioxidant, analgesic and anti-inflammatory activities of extracellular polysaccharide of nostoc flagelliforme under Salt Stress. Food Science 41(17):133-138.

6. Chen C Y, Department S. 2016. Determination of Anthocyanins in Purple Sweet Potato by Microwave Digestion and Visible Spectrophotometric. Food Research and Development, 37(4): 158-160.

7. Chen X, Mao X, Huang J, Ding Y, Wu J, Dong S, Kong L, Gao G, Li CY, Wei L. 2011. KOBAS Research 39:316-322.

10. Fu X, Zou Z. 2015. Abiotic regulation: A common way for proteins to modulate their functions. Current Protein and Peptide Science 16(3): 188-195.

11. Gangappa S N, Maurya J P, Yadav V, Chattopadhyay S. 2013. The regulation of the Z- and G-box containing promoters by light signaling components, SPA1 and MYC2, in Arabidopsis. Plos One 8(4):7377-7382.

12. Gao L, Li Y , Han R. 2016. Cell wall reconstruction and DNA damage repair play a key role in the 
547

548

549

550

551

552

553

554

555

556

557

558

559

560

561

562

563

564

565

566

567

improved salt tolerance effects of He-Ne laser irradiation in tall fescue seedlings. Bioscience, Biotechnology, and Biochemistry 80: 682-693.

13. Hamann. 2015. The plant cell wall integrity maintenance mechanism-A case study of a cell wall plasma membrane signaling network. Phtyochemistry 112:100-109.

14. Huang X, Ouyang X, Deng X W. 2014. Beyond repression of photomorphogenesis: role switching of COP/DET/FUS in light signaling. Current Opinion in Plant Biology 21:96-103.

15. Ingram G, Nawrath C. 2017. The roles of the cuticle in plant development: organ adhesions and beyond. Journal of Experimental Botany 68(19):5307-5321.

16. Jacob P, Hirt H, Bendahmane A. 2017. The heat-shock protein/chaperone network and multiple stress resistance. Plant Biotechnology Journal 15(4):405-414.

17. Kriechbaumer V, Brandizzi F. 2020. The plant endoplasmic reticulum: an organized chaos of tubules and sheets with multiple functions. Journal of Microscopy 280(2):122-133.

18. Lan XG, Yang J, Abhinandan K, Nie YZ, Li XY, Li YH, Samuel MA. 2017. Flavonoids and ROS play opposing roles in mediating pollination in Ornamental Kale (Brassica oleracea var. acephala). Molecular Plant (Cell Press) 10(10):1361-1364.

19. Leivar P, Tepperman JM, Cohn M M, Monte E, Al-sady B, Erickson E, Quail PH. 2012. Dynamic antagonism between phytochromes and PIF family basic Helix-Loop-Helix factors induces selective reciprocal responses to light and shade in a rapidly responsive transcriptional network in Arabidopsis. Plant Cell 24(4):1398-1419.

20. Li H, Ye W, Wang Y, Chen XH, Fang Y, Sun G. 2021. RNA sequencing-based exploration of the effects of far-red light on lncRNAs involved in the shade-avoidance response of D. officinale. PeerJ 
9(1):e10769.

569

570

571

572

573

574

575

576

577

578

579

580

581

582

583

584

585

586

587

21. Li H, Lyu Y, Chen X, Wang CQ, Yao DH, Ni SS, Lin YL, Chen YK, Zhang ZH, Lai, ZX. 2019. Exploration of the effect of blue light on functional metabolite accumulation in longan embryonic calli via RNA sequencing. International Journal of Molecular Sciences 20(2):441-464.

22. Li YF, Gao LM, Han R. 2016. A combination of He-Ne laser irradiation and exogenous nitric oxide application efficiently protect wheat seedling from oxidative stress caused by elevated UV-B stress. Environmental Science and Pollution Research 23: 23675-23682.

23. Lin J B, Wang W Y, Zou H, Dai YM. 2019. Transcriptome analysis on pathway of and genes related to flavonoid synthesis in Dendrobium officinale. Fujian Journal of Agricultural Sciences 34(9):1019-1025.

24. Lin X, Lai Z. 2015. Effect of light quality on the proliferation of protocorm and active ingredient contents of Dendrobium officinale. Chinese Journal of Tropical Crops 36 (10): 1796-1801.

25. Liu H, Wang Q, Liu Y, Zhao XY, Imaizumi T, Somers DE, Toin EM, Lin C. 2013. Arabidopsis CRY2 and ZTL mediate blue-light regulation of the transcription factor CIB1 by distinct mechanisms. Proceedings of the National Academy of Sciences of the United States of America 110(43):17582-17587.

26. Liu X, Sun H, Chen W, Guo Q, Xiaolin LI, Liang G. 2016. Advances in studies on carotenoids in loquat fruit. Journal of Fruit Science 35(6): 932-939.

27. Liu Y, He C. 2016. Regulation of plant reactive oxygen species (ROS) in stress responses: learning from AtRBOHD. Plant Cell Reports 35(5):995-1007.

28. Liu Y, Patra B, Pattanaik S, Wang Y, Yuan L. 2019. GATA and PIF transcription factors regulate light-induced vindoline biosynthesis in Catharanthus roseus. Plant Physiology 180(3):1336-1350. 
588

589

590

591

592

593

594

595

596

597

598

599

600

601

602

603

604

605

606

607

608

29. Liu Z, Zhang Y, Wang J, Li P, Zhao C, Chen Y, Bi Y. 2015. Phytochrome-interacting factors PIF4 and PIF5 negatively regulate anthocyanin biosynthesis under red light in Arabidopsis seedlings. Plant Science 238:64-72.

30. Luijtelaar A V, Greenwood B M, Ahmed H U, Barqawi AB, Barret E. 2019. Focal laser ablation as clinical treatment of prostate cancer: report from a Delphi consensus project. Springer Open Choice 37(10): $2147-2153$.

31. Luo L, Xu XH, Yang K, Li Z, Zhang XQ. 2020. Senescence and heat shock protein in plants in response to abiotic stress. Pratacultural Science 37(11): 2320-2333.

32. Martin C, Zhang Y, Stefano RD, Robine M, Butelli E, Bulling K, Hill L, Rejzek M, Martin C, Schoonbeek HJ. 2015. Different ROS-scavenging properties of flavonoids determine their abilities to extend shelf life of tomato. Free Radical Biology \& Medicine the Official Journal of the Oxygen Society 169:1568-1583.

33. Martins I, Hartmann D O, Alves P C, Martins C, Garcia H, Leclercq CC, Ferreira R, He J, Renaut J, Becker JD, Pereira CS. 2014. Elucidating how the saprophytic fungus Aspergillus nidulans uses the plant polyester suberin as carbon source. BMC Genomics 15: 613-632.

34. Meng Y, Lin C. 2013. Blue Light-dependent interaction between Cryptochrome 2 and CIB1 regulates transcription and leaf senescence in Soybean. Plant Cell 25(11):4405-4420.

35. Morales-Cedillo F, González-Solís A, Gutiérrez-Angoa L, Cano-Ramírez DL, Gavilanes-Ruiz M. 2015. Plant lipid environment and membrane enzymes: the case of the plasma membrane H+-ATPase. Plant Cell Reports 34(4):617-629.

36. Nhut DT, Huy NP, Tai NT, Nam NB, Luan VQ, Hien VT, Tung HT, Vinh BT, Luan CT. 2015. 
609

610

611

612

613

614

615

616

617

618

619

620

621

622

624

625

626

627

628

629

Light-emitting diodes and their potential in callus growth, plantlet development and saponin accumulation during somatic embryogenesis of Panax vietnamensis Ha et Grushv. Biotechnology \& Biotechnological Equipment 29(2):299-308.

37. Satoh M, Tokaji Y, Nagano A J, Hara-Nishimura I, Hayashi M, Nishimura M, Ohta H, Masuda S. 2014. Arabidopsis mutants affecting oxylipin signaling in photo-oxidative stress responses. Plant Physiology \& Biochemistry 81:90-95.

38. Savchenko TV, Zastrijnaja OM, Klimov VV. 2014. Oxylipins and plant abiotic stress resistance. Biochemistry 79(4):362-375.

39. Sethi V, Raghuram B, Sinha A K, Chattopadhyay S. 2014. A mitogen-activated protein kinase cascade module, MKK3-MPK6 and MYC2, is involved in blue light-mediated seedling development in Arabidopsis. Plant Cell 26(8):3343-3357.

40. Shen C, Guo H, Chen H, Shi Y, Meng Y, Lu J, Feng S, Wang H. 2017. Identification and analysis of genes associated with the synthesis of bioactive constituents in Dendrobium officinale using RNA-Seq. Scientific Reports, 7(1):187.

41. Shi H, Liu R, Xue C, Shen X, Wei N, Deng XW, Zhong S. 2016. Seedlings transduce the depth and mechanical pressure of covering soil using $C O P 1$ and ethylene to regulate $E B F 1 / E B F 2$ for soil emergence. Current Biology 26: 139-149.

42. Su J, Liu B, Liao J, Yang Z, Lin C, Oka Y. 2017. Coordination of cryptochrome and phytochrome signals in the regulation of plant light responses. Agronomy 7(1):25.

43. Tang L, Wang C Y, Long H, Li J, Chen GX, Zhou Q. 2019. Effects of environmental factors on growth and development and effective component contents in Dendrobium officinale. Journal of Chinese 
630

631

632

633

634

635

636

637

638

639

640

641

642

643

644

645

646

647

648

649

650

44. Vishwanath S J, Delude C, Domergue F, Rowland O. 2015. Suberin: biosynthesis, regulation, and polymer assembly of a protective extracellular barrier. Plant Cell Reports 34(4):573-586.

45. Wan WC, Shi MM, Zhang B. 2020. Development of plant laser lamp. Journal of Technology 20(1): 3339.

46. Wang P, Hawes C, Hussey P J. 2017. Plant endoplasmic reticulum-plasma membrane contact sites. Trends in Plant Science 22(4):289-297.

47. Wang Q, Barshop W D, Bian M, Vashisht AA, He R, Yu X, Liu B, Nguyen P, Liu X, Zhao X, Wohlschlegel JA, Lin C. 2015. The blue light-dependent phosphorylation of the CCE domain determines the photosensitivity of Arabidopsis CRY2. Molecular plant 8(4):631-643.

48. Wang W T, Zhang J W, Wang D, Tao SH, Ji YL, Wu B. 2010. Relation between light qualities and accumulation of steroidal glycoalkaloids as well as signal molecule in cell in potato tubers. Acta Agronomica Sinica 36(4):629-635.

49. Xu L, Liu L, Peng SD, Li WY, Zhang T, Li ZM, Wang HM, Li SJ, Lin LG. 2015. Genetic diversity of Dendrobium officinale revealed by SSR markers. Molecular Plant Breeding (7): 1616-1622.

50. Yang Z, Liu B, Su J, Liao JK, Lin C, Oka Y. 2017. Cryptochromes orchestrate transcription regulation of diverse blue light responses in plants. Photochemistry \& Photobiology 93(1):112-127.

51. Yeung K, Ortner V K, Martinussen T, Paasch U. 2019. Efficacy of laser treatment for onychomycotic nails: a systematic review and meta-analysis of prospective clinical trials. Lasers in Medical Science 34(4): 1513-1525.

52. Young MD, Wakefield M J, Smyth G K,Oshlack A. 2020. Gene ontology analysis for RNA-seq: 

accounting for selection bias. Genome Biology 11(2):14-R14.

652

653

654

655

656

657

658

659

660

661

53. Zhang GQ, Xu Q, Bian C, Tsai WC, Yeh CM. 2016. The Dendrobium catenatum Lindl, genome sequence provides insights into polysaccharide synthase, floral development and adaptive evolution. Scientific Reports 6(19029):1-10.

54. Zhang H, Hedhili S, Montiel G, Zhang Y, Chatel G, Pré M, Gantet P, Memelink J. 2011. The basic helix-loop-helix transcription factor $C r M Y C 2$ controls the jasmonate-responsive expression of the $O R C A$ genes that regulate alkaloid biosynthesis in Catharanthus roseus. Plant Journal for Cell \& Molecular Biology 67(1):61-71.

55. Zhang QZ, Wu NY, Jian DQ, Jiang R, Yang C, Lan X, Chen M, Zhan F, Liao Z. 2019. Overexpression of AaPIF3 promotes artemisinin production in Artemisia annua. Industrial Crops and Products 138:111476-111488. 
Figure 1

Figure 1 The phenotype of $D$. officinale under different light treatments.

A-C represent the phenotype before white, blue and blue laser light treatments respectively.

D-F represent the phenotype after white, blue and blue laser light treatments respectively.

Bars $=10 \mathrm{~mm}$.

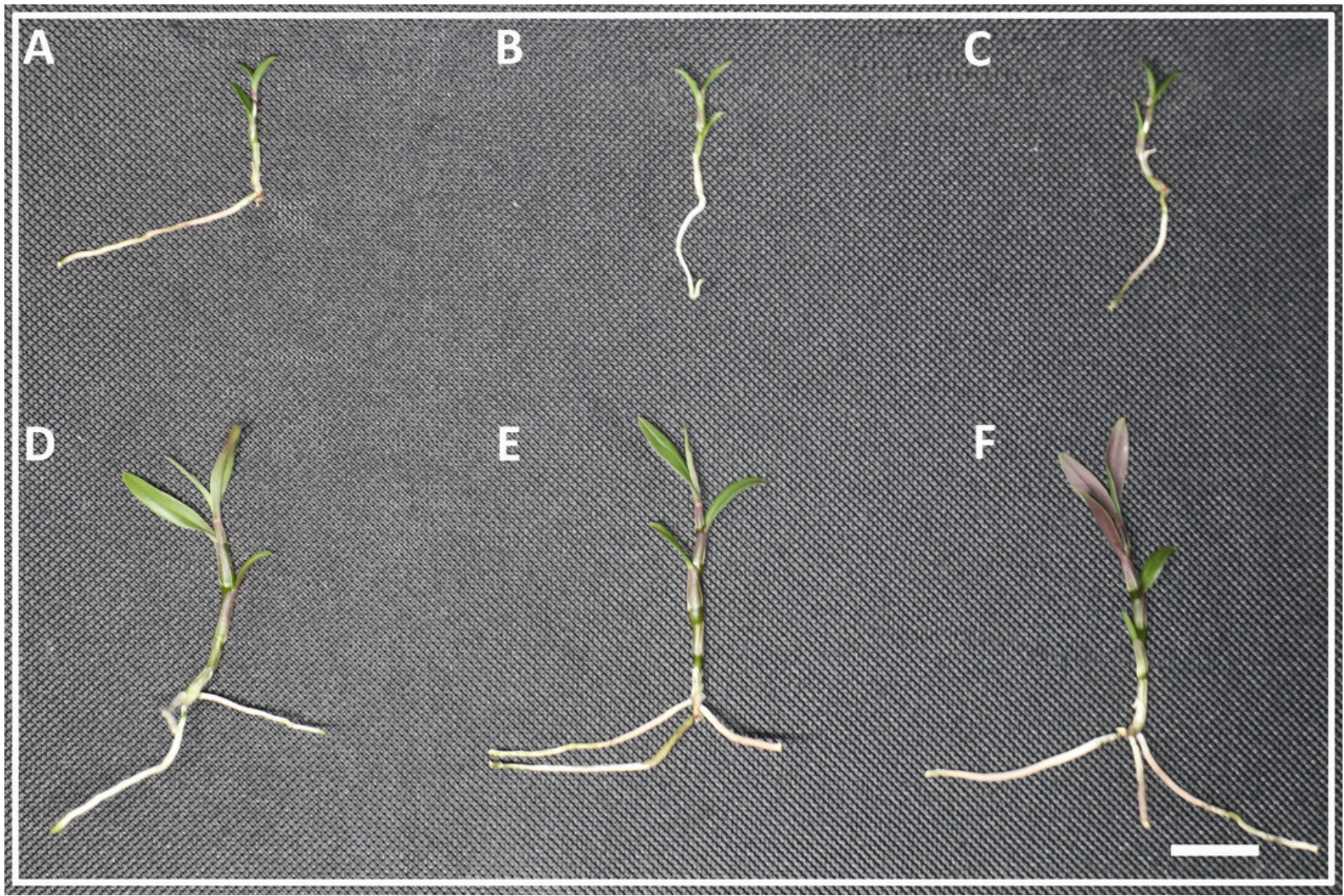


Figure 2

Figure 2 Differentially expressed genes under the different light treatments.

A, Number of genes with low $($ FPKM $<5)$, moderate $(5 \leq$ FPKM $\leq 50)$ and high $(F P K M>50)$ expression in each library. B, Venn diagram showing the numbers of unique and commonly regulated genes identified from the LB-B, LB-W and B-W comparisons. C, DEGs in response to different light treatments.
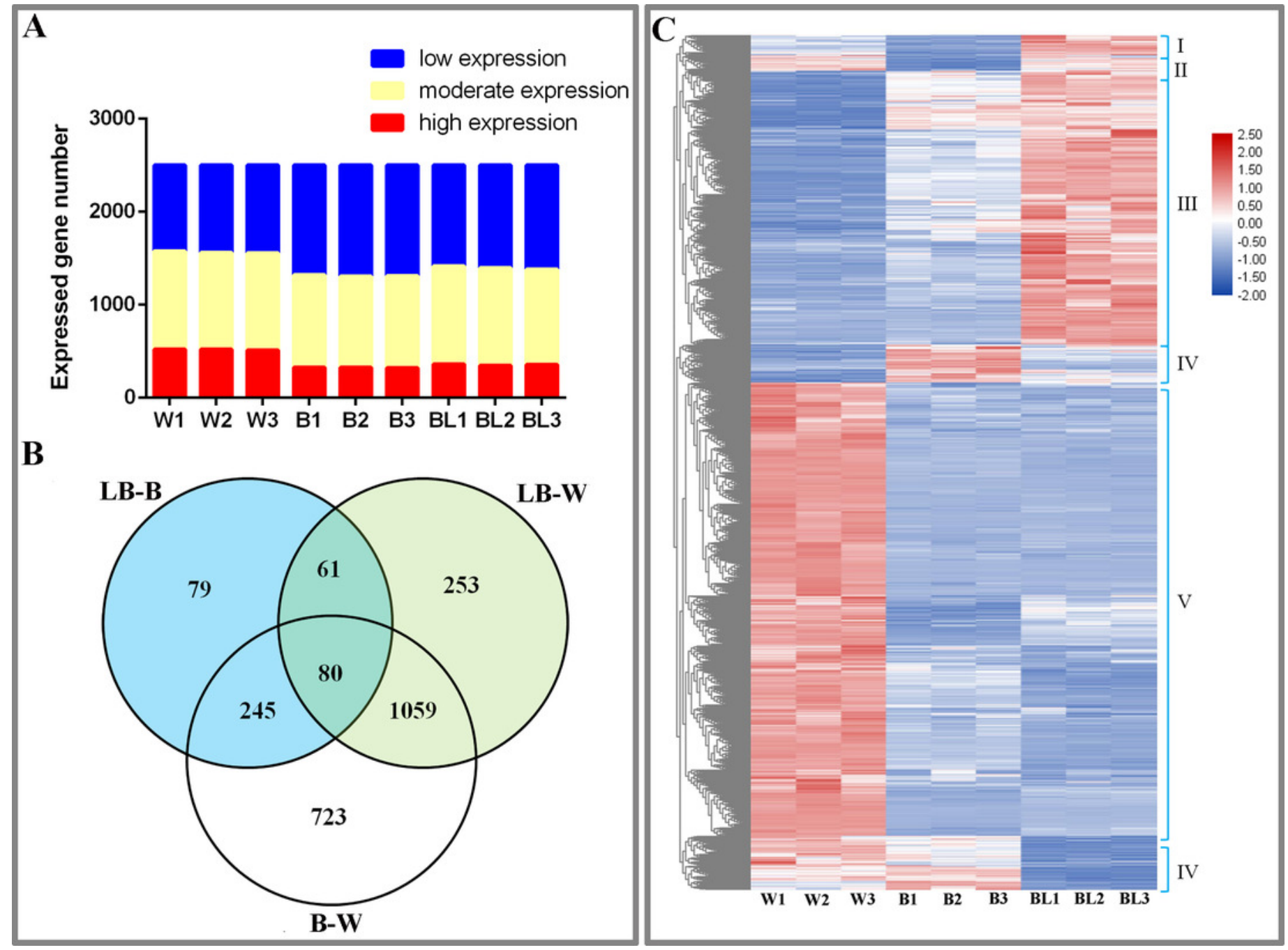


\section{Figure 3}

Figure 3 KEGG enrichment analysis of DEGs in D. officinale under different light treatments.

A, LB-B; B, LB-W. C, B-W; D, Top 20 KEGG pathways enriched in DEGs in the three groups. The red colour indicates that the comparison contains the pathway, and the blue colour indicates that the comparison does not contain the pathway.

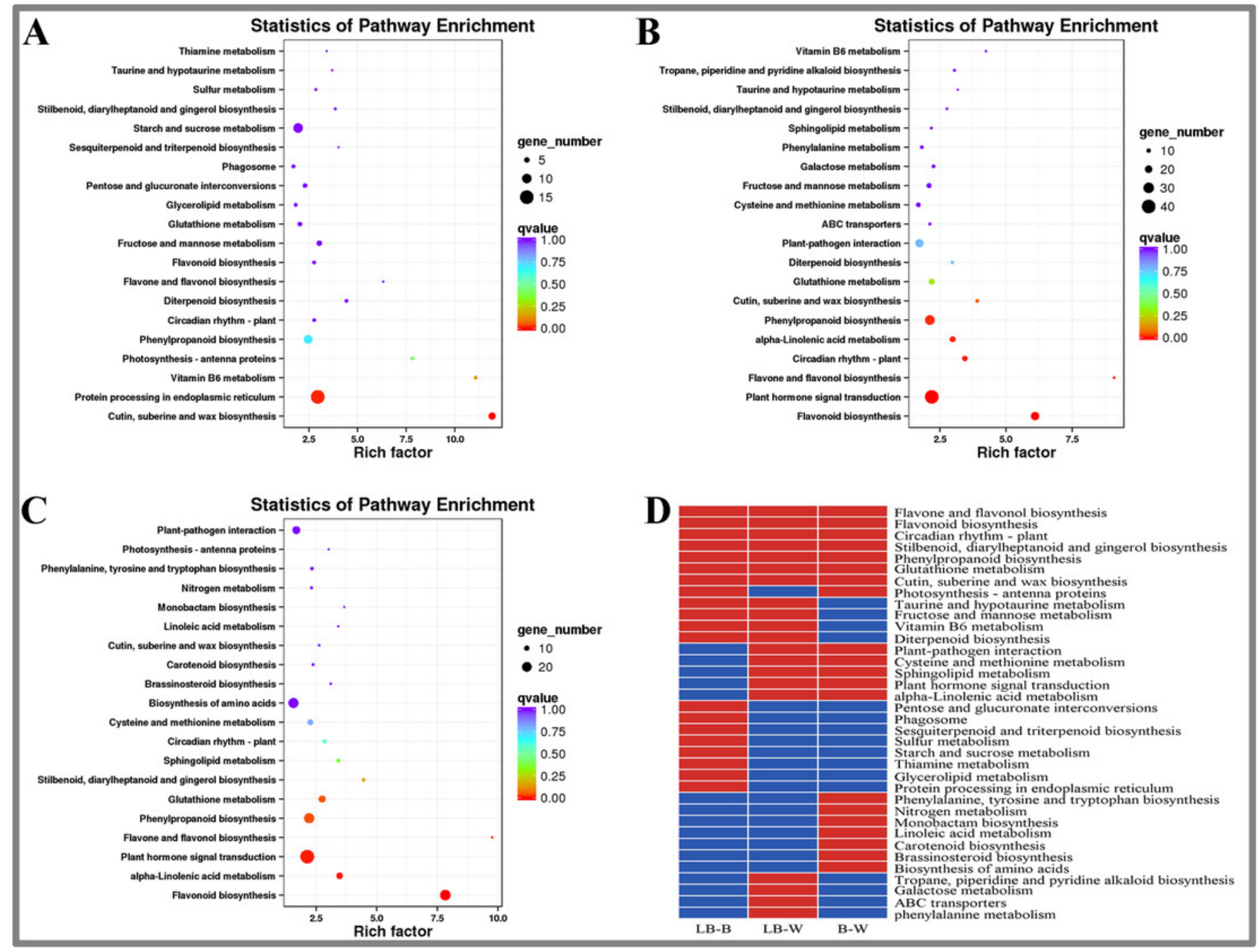


Figure 4

Figure 4 Levels of physiological and biochemical indicators in the leaves of $D$. officinale under different light treatments.

A, POD activity; B, SOD activity; C, PAL activity. Different upper/lowercase letters indicate statistically significant differences at the $0.01 / 0.05$ level, as determined by one-way ANOVA and Duncan's test.

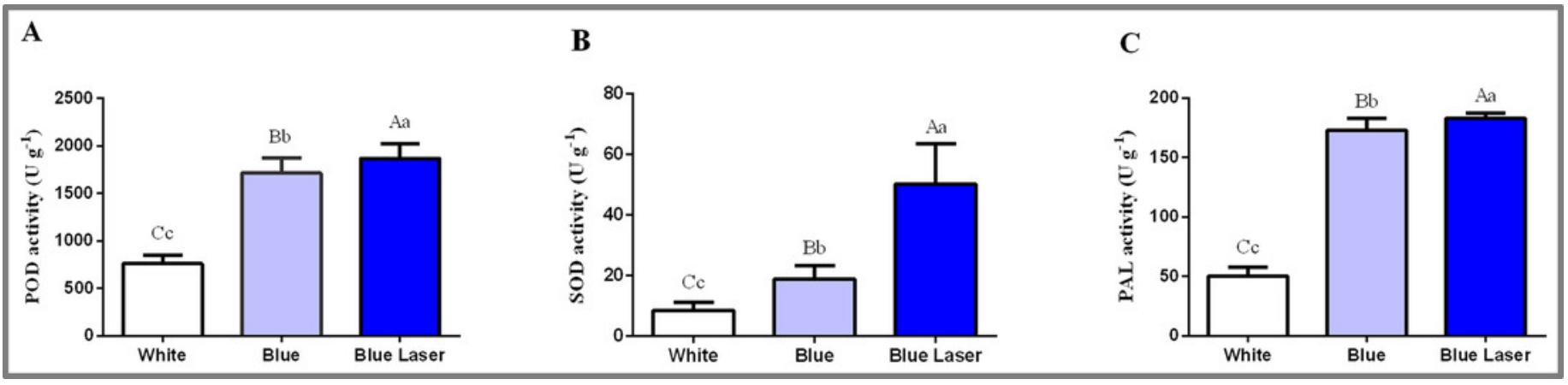




\section{Figure 5}

Figure $5 \mathrm{DE}$ metabolic pathway synthetic genes and functional metabolite contents in $D$. officinale under different light treatments.

$A, F, I$ represent FPKM value changes in DE synthetic genes of flavonoid metabolic pathway, polysaccharide metabolic pathway and alkaloid metabolic pathway respectively. B and C represent changes in the flavonoid contents in the leaves and stems respectively. D and $E$ represent changes in the anthocyanin contents in the leaves and stems respectively. $\mathrm{G}$ and $\mathrm{H}$ represent changes in the polysaccharide contents in the leaves and stems respectively. J and $\mathrm{K}$ represent changes in the alkaloid contents in the leaves and stems respectively. 

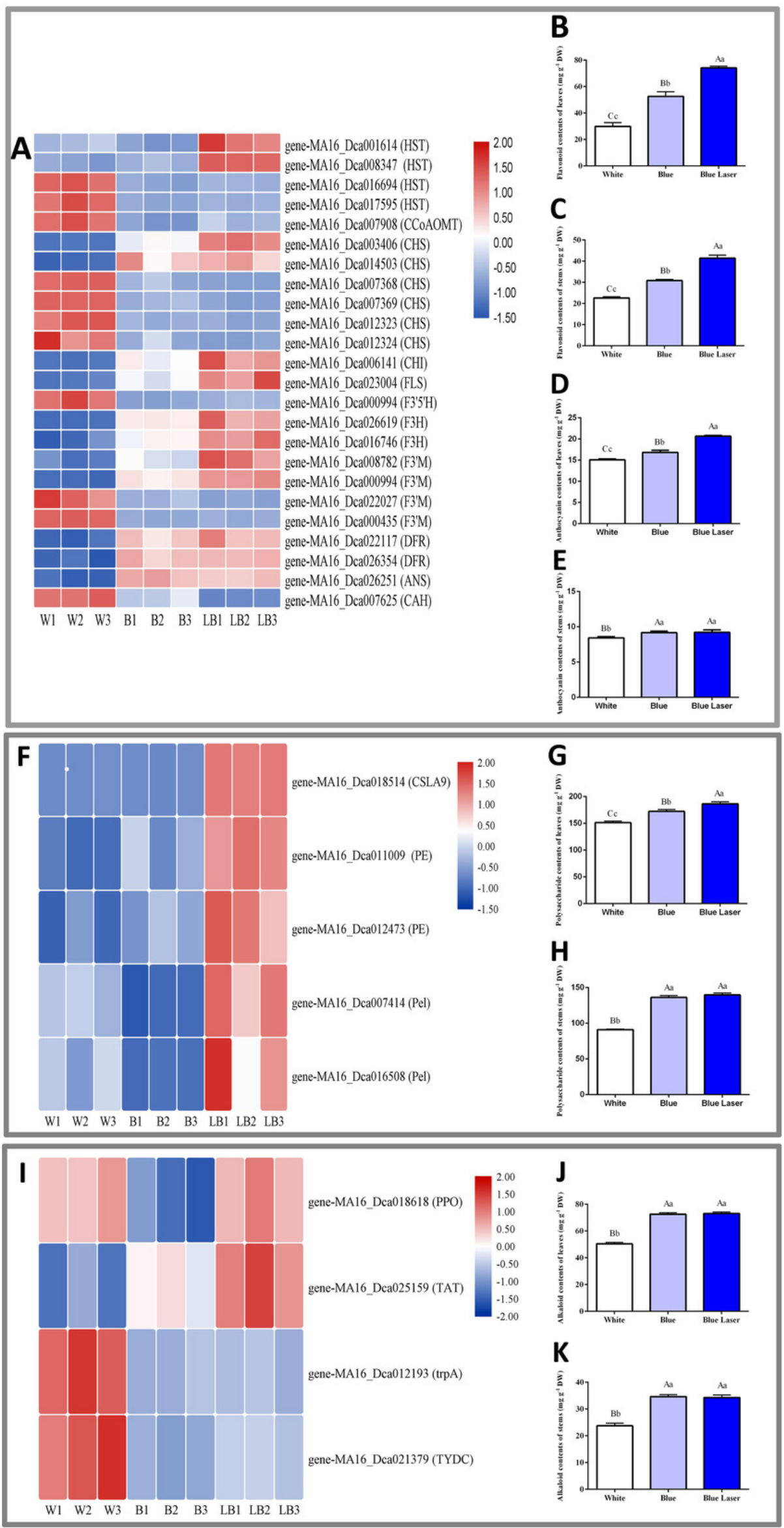
Figure 6

Figure 6 qPCR verification of DEGs in D. officinale under different light treatments.

HSP70, heat shock cognate $70 \mathrm{kDa}$ protein 2-like; CYP86A4S, fatty acid omega-hydroxylase; ERF13, ethylene response factor 13; CRY DASH, cryptochrome dash; SPA1, phytochrome A suppressor 1; HY5, long hypocotyl 5; CO16, constants 16; MYC2, basic helix-loop-helix transcription factor; PIF4, Phytochrome-interacting factor 4.

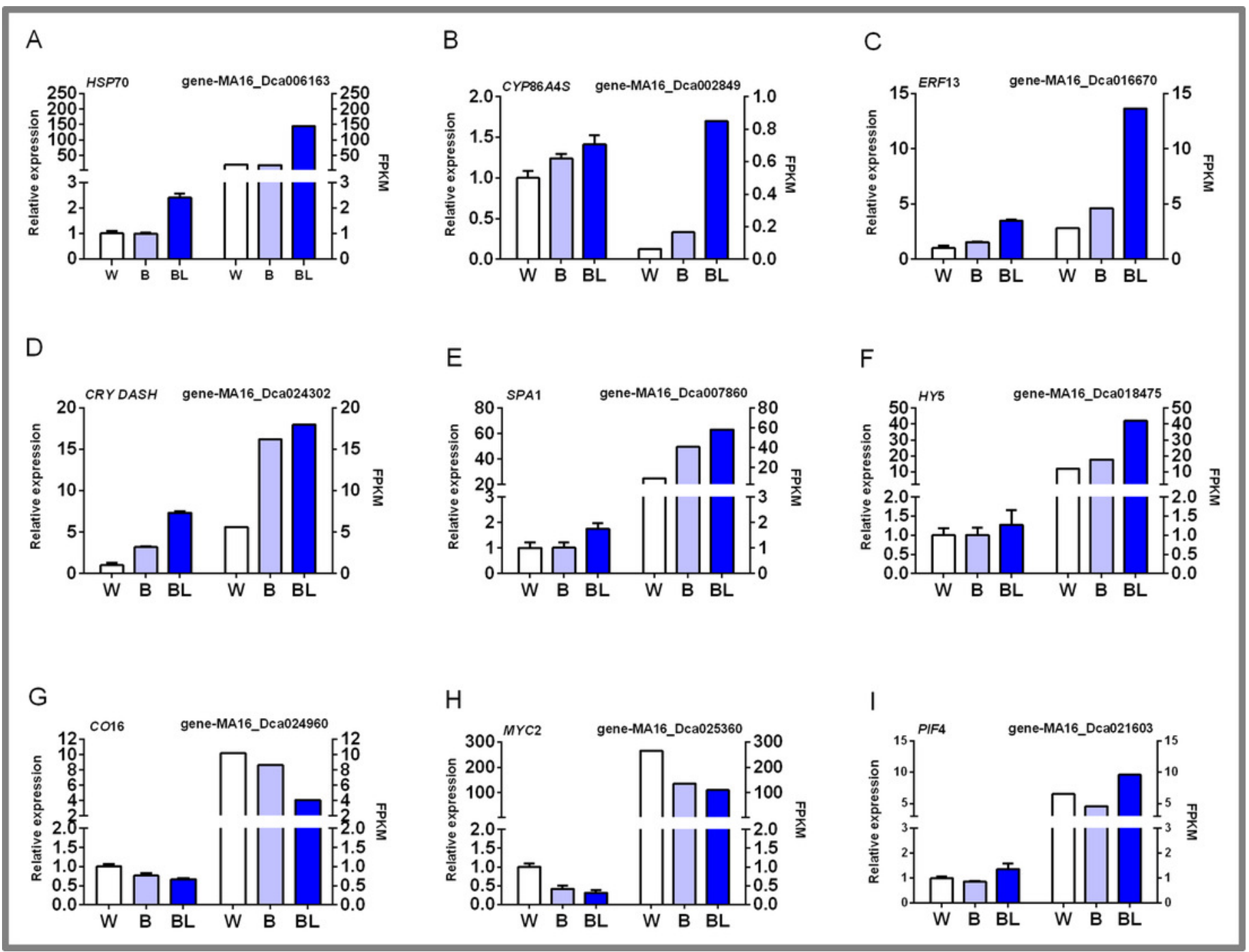


Figure 7

Figure $7 \mathrm{~A}$, Blue laser signal network of functional metabolites in $D$. officinale. B, Heat maps of the blue laser signaling networks in up- and downregulated genes.

Red font indicates differentially expressed genes. 

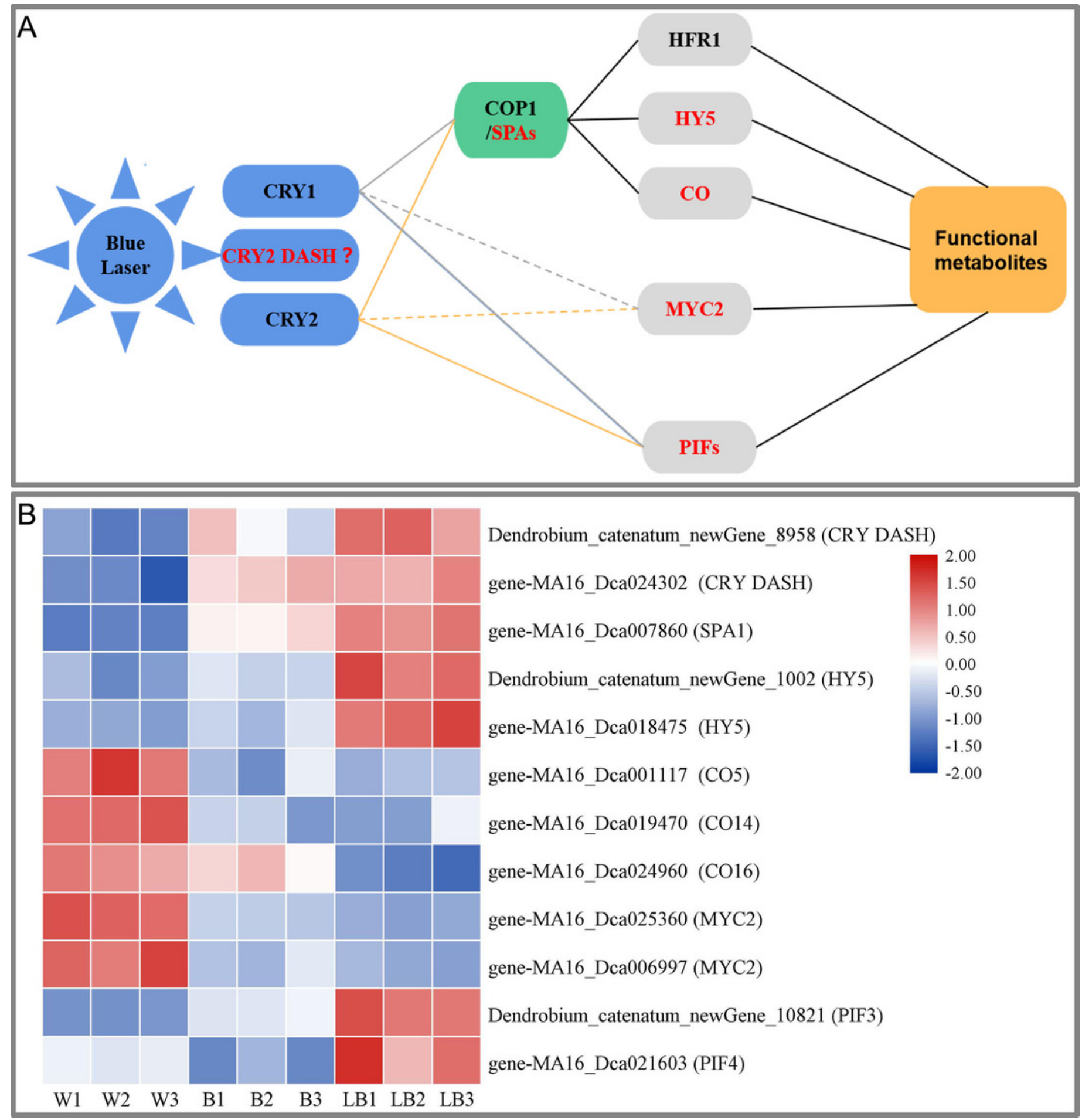


\section{Table $\mathbf{1}$ (on next page)}

Table 1 mRNA results from nine $D$. officinale libraries 
Table 1 mRNA results from nine $D$. officinale libraries

\begin{tabular}{cccccc}
\hline Samples & Total reads & Total mapped reads (\%) & $\begin{array}{c}\text { Uniquely mapped } \\
\text { reads (\%) }\end{array}$ & $\begin{array}{r}\text { Q30 } \\
(\%)\end{array}$ & GC content (\%) \\
\hline W1 & $50,264,012$ & 88.63 & 86.20 & 92.53 & 46.07 \\
W2 & $53,636,818$ & 88.86 & 86.56 & 92.75 & 46.13 \\
W3 & $54,223,452$ & 88.97 & 86.42 & 92.47 & 46.03 \\
B1 & $45,129,776$ & 89.02 & 86.70 & 93.93 & 46.02 \\
B2 & $40,640,606$ & 88.54 & 86.17 & 93.72 & 45.95 \\
B3 & $42,995,976$ & 87.96 & 85.70 & 93.41 & 45.91 \\
LB1 & $41,961,634$ & 88.76 & 86.44 & 93.12 & 45.83 \\
LB2 & $50,932,878$ & 88.42 & 86.10 & 92.54 & 45.78 \\
LB3 & $42,002,508$ & 88.40 & 86.16 & 92.63 & 45.86 \\
\hline
\end{tabular}

2 
Table 2 (on next page)

Table 2 Top five GO terms obtained from an enrichment analysis of DEGs in $D$. officinale under different light treatments 
1 Table 2 Top five GO terms obtained from an enrichment analysis of DEGs in $D$. officinale under different light

2 treatments

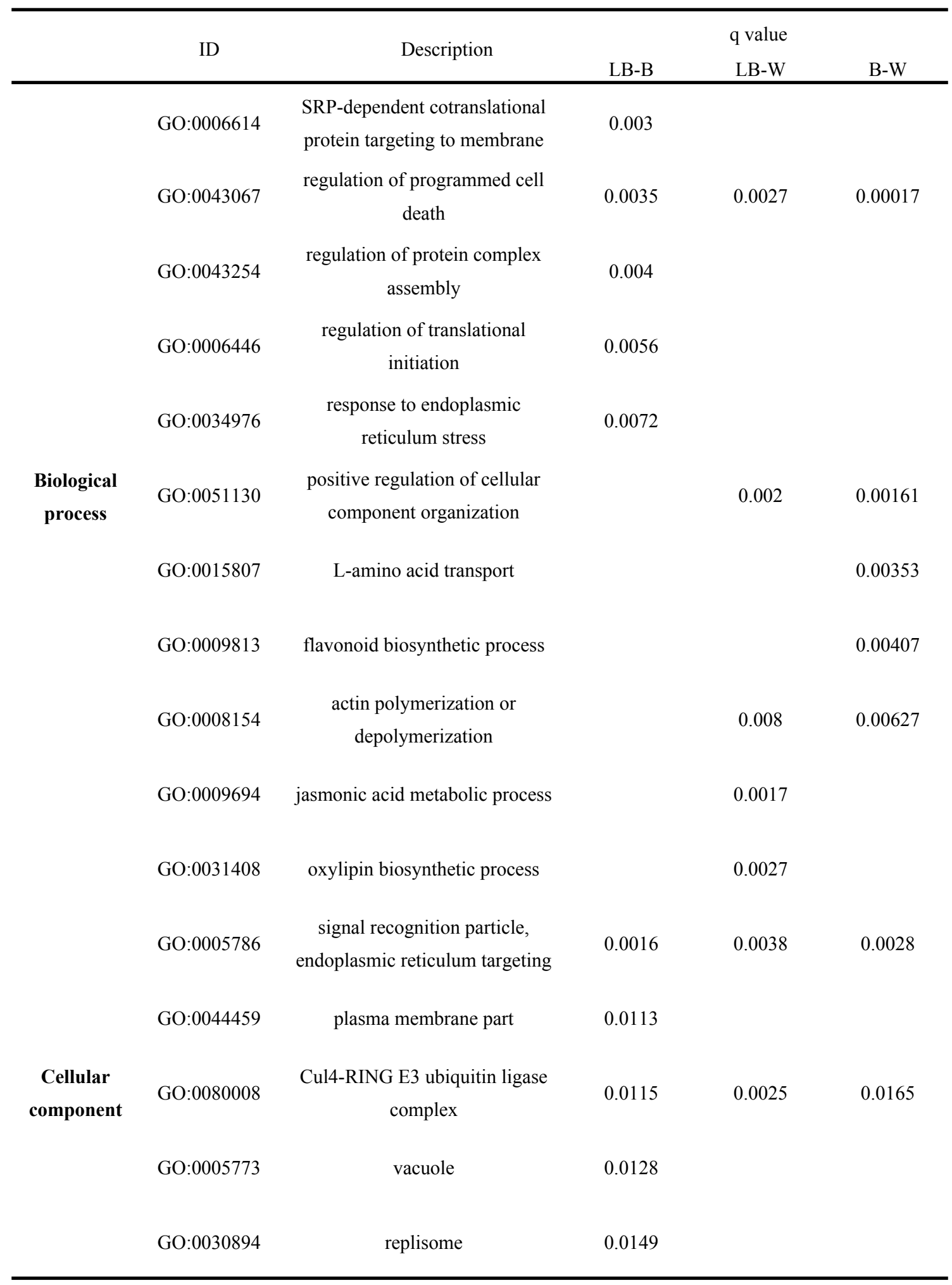




\begin{tabular}{|c|c|c|c|c|c|}
\hline & GO:0031226 & $\begin{array}{l}\text { intrinsic component of plasma } \\
\text { membrane }\end{array}$ & & & 0.0017 \\
\hline & GO:0016021 & integral component of membrane & & 0.0033 & 0.0043 \\
\hline & GO:0005887 & $\begin{array}{l}\text { integral component of plasma } \\
\text { membrane }\end{array}$ & & 0.0091 & 0.0174 \\
\hline & GO:0005618 & cell wall & & 0.0148 & \\
\hline & GO:0008312 & 7S RNA binding & 0.00044 & 0.00183 & 0.00119 \\
\hline \multirow{6}{*}{$\begin{array}{l}\text { Molecular } \\
\text { function }\end{array}$} & GO:0016702 & $\begin{array}{l}\text { oxidoreductase activity, acting } \\
\text { on single donors with } \\
\text { incorporation of molecular } \\
\text { oxygen, incorporation of two } \\
\text { atoms of oxygen }\end{array}$ & 0.00064 & 0.00111 & 0.00174 \\
\hline & GO:0051082 & unfolded protein binding & 0.00138 & & \\
\hline & GO:0016705 & $\begin{array}{l}\text { oxidoreductase activity, acting } \\
\text { on paired donors, with } \\
\text { incorporation or reduction of } \\
\text { molecular oxygen }\end{array}$ & 0.00438 & 0.00012 & 0.00062 \\
\hline & GO:0003964 & $\begin{array}{c}\text { RNA-directed DNA polymerase } \\
\text { activity }\end{array}$ & 0.00536 & & \\
\hline & GO:0016210 & $\begin{array}{l}\text { naringenin-chalcone synthase } \\
\text { activity }\end{array}$ & & 0.00056 & 0.0003 \\
\hline & GO:0005506 & iron ion binding & & 0.00032 & 0.00033 \\
\hline
\end{tabular}

3 


\section{Table 3 (on next page)}

Table 3 The top 10 up-and down-regulation DEGs in the LB-B 


\begin{tabular}{|c|c|c|c|c|c|}
\hline NO & Gene ID & gene_name & $\begin{array}{c}\quad \log _{2} \\
\text { (Laser/Blue) }\end{array}$ & regulated & NR_annotation \\
\hline 1 & gene-MA16_Dca014371 & CLPB1 & 2.946278 & up & chaperone protein $\mathrm{ClpB} 1$ \\
\hline 2 & gene-MA16_Dca006163 & HSP70 & 2.937094 & up & heat shock cognate $70 \mathrm{kDa}$ protein 2-like \\
\hline 3 & gene-MA16_Dca000936 & $\mathrm{INV} * \mathrm{DC} 4$ & 2.902941 & up & Beta-fructofuranosidase, soluble isoenzyme I \\
\hline 4 & gene-MA16_Dca018514 & CSLA9 & 2.900806 & up & Glucomannan 4-beta-mannosyltransferase 9 \\
\hline 5 & gene-MA16_Dca013846 & HSP23 & 2.662168 & up & small heat shock protein, chloroplastic-like \\
\hline 6 & gene-MA16_Dca026995 & HSP18.6 & 2.516762 & up & $18.6 \mathrm{kDa}$ class III heat shock protein \\
\hline 7 & gene-MA16_Dca018083 & HSP83A & 2.485551 & up & heat shock protein 83 \\
\hline 8 & gene-MA16_Dca001614 & CCOMT & 2.662168 & up & small heat shock protein, chloroplastic-like \\
\hline 10 & gene-MA16_Dca001256 & ABCG11 & 2.485551 & up & heat shock protein 83 \\
\hline 1 & gene-MA16_Dca003810 & TSPO & -2.9122 & down & translocator protein homolog \\
\hline 2 & gene-MA16_Dca022104 & CAB3C & -2.90546 & down & chlorophyll a-b binding protein of LHCII type 1-like \\
\hline 3 & gene-MA16_Dca024558 & PETE & -2.35939 & down & plastocyanin-like \\
\hline 4 & gene-MA16_Dca017227 & XERO1 & -2.27399 & down & Dehydrin Xero 1 \\
\hline 5 & gene-MA16_Dca008991 & CYP81E1 & -2.12773 & down & Isoflavone 2\&apos;-hydroxylase \\
\hline 6 & gene-MA16_Dca009695 & HT1 & -2.00848 & down & $\begin{array}{l}\text { serine/threonine-protein kinase STY46-like isoform } \\
\mathrm{X} 2\end{array}$ \\
\hline 7 & gene-MA16_Dca015158 & CYP71A1 & -2.00817 & down & Cytochrome P450 71A1 \\
\hline 8 & gene-MA16_Dca016021 & GMPM1 & -1.99738 & down & $11 \mathrm{kDa}$ late embryogenesis abundant protein-like \\
\hline 9 & gene-MA16_Dca002504 & SAT2 & -1.93201 & down & probable serine acetyltransferase 2 isoform $\mathrm{X} 1$ \\
\hline 10 & gene-MA16_Dca008990 & CYP81E1 & -1.927 & down & Isoflavone 2\&apos;-hydroxylase \\
\hline
\end{tabular}

2 
Table 4 (on next page)

Table 4 The top 10 up-and down-regulation DEGs in the LB-W 


\begin{tabular}{|c|c|c|c|c|c|}
\hline NO & Gene ID & gene_name & $\begin{array}{c}\log _{2} \\
\text { (Laser/Blue) }\end{array}$ & regulated & NR_annotation \\
\hline 1 & gene-MA16_Dca024845 & ELIP1 & 5.335425 & up & $\begin{array}{l}\text { early light-induced protein } 1 \text {, } \\
\text { chloroplastic-like }\end{array}$ \\
\hline 2 & gene-MA16_Dca003829 & $\mathrm{C} 1$ & 5.219541 & up & trichome differentiation protein GL1-like \\
\hline 3 & gene-MA16_Dca022385 & RALFL33 & 4.06727 & up & Protein RALF-like 33 \\
\hline 4 & gene-MA16_Dca003827 & $\mathrm{C} 1$ & 3.990023 & up & transcription factor MYB114-like \\
\hline 5 & gene-MA16_Dca001256 & ABCG11 & 3.550505 & up & $\mathrm{ABC}$ transporter $\mathrm{G}$ family member 11 -like \\
\hline 6 & gene-MA16_Dca021634 & MAN6 & 3.505578 & up & mannan endo-1,4-beta-mannosidase 6-like \\
\hline 7 & gene-MA16_Dca005399 & APG & 3.429596 & up & GDSL esterase/lipase APG \\
\hline 8 & gene-MA16_Dca018083 & HSP83A & 3.384163 & up & heat shock protein 83 \\
\hline 9 & gene-MA16_Dca012014 & NIP2-1 & 3.344414 & up & aquaporin NIP2-1-like \\
\hline 10 & gene-MA16_Dca026995 & HSP18.6 & 3.318636 & up & $18.6 \mathrm{kDa}$ class III heat shock protein \\
\hline 1 & gene-MA16_Dca015278 & CYP94A1 & -7.75405 & down & cytochrome P450 94B3-like \\
\hline 2 & gene-MA16_Dca015280 & CYP94A1 & -7.31088 & down & cytochrome P450 94B1-like \\
\hline 3 & gene-MA16_Dca022981 & SRG1 & -7.01481 & down & $\begin{array}{l}\text { probable 2-oxoglutarate-dependent dioxygenase } \\
\text { At5g05600 }\end{array}$ \\
\hline 4 & gene-MA16_Dca017290 & ABCG11 & -6.7603 & down & $\mathrm{ABC}$ transporter $\mathrm{G}$ family member 11 \\
\hline 5 & gene-MA16_Dca017057 & ERF112 & -6.4656 & down & Ethylene-responsive transcription factor ERF112 \\
\hline 6 & gene-MA16_Dca008990 & CYP81E1 & -6.2956 & down & Isoflavone 2\&apos;-hydroxylase \\
\hline 7 & gene-MA16_Dca008681 & ERF114 & -6.06792 & down & $\begin{array}{l}\text { ethylene-responsive transcription factor ERF110- } \\
\text { like }\end{array}$ \\
\hline 8 & gene-MA16_Dca014747 & CBSX1 & -5.90332 & down & $\begin{array}{l}\text { CBS domain-containing protein CBSX1, } \\
\text { chloroplastic-like }\end{array}$ \\
\hline 9 & gene-MA16_Dca026750 & CYP735A1 & -5.82538 & down & Cytokinin hydroxylase \\
\hline 10 & gene-MA16_Dca015945 & FLS & -5.82253 & down & $\begin{array}{l}\text { probable 2-oxoglutarate-dependent dioxygenase } \\
\text { At5g05600 }\end{array}$ \\
\hline
\end{tabular}

